\title{
Successful Removal of Cranial Migrated Intravitreal Perfluorocarbon through A Lumbar Puncture
}

\author{
Lin Chen \\ Jinhua Municipal Central Hospital \\ Zhongheng Zhang \\ Zhejiang University School of Medicine \\ Qing Wang \\ University of Virginia \\ Hongying Ni ( $\nabla$ nihongying2@163.com ) \\ Jinhua Municipal Central Hospital
}

Case report

Keywords: Perfluorocarbon, Lumbar puncture

Posted Date: January 22nd, 2020

DOI: https://doi.org/10.21203/rs.2.21567/v1

License: (9) This work is licensed under a Creative Commons Attribution 4.0 International License.

Read Full License 


\section{Abstract}

Background: Perfluorocarbon migrating into subarachnoid was very rare let alone its further removing from central nervous system.

Case presentation: We report a case of migration of intravitreal perfluorocarbon into cranial space and further removed through a lumbar puncture treatment. The patient showed a sudden onset of hypoxemia and hypotension during an ocular surgery, which was highly suspected as neurogenic pulmonary edema caused by the migrated perfluorocarbon. The patient's symptoms improved after extracorporeal membrane oxygenation (ECMO). The perfluorocarbon gradually descended through subarachnoid space to lumbar cisterna and finally drained by a lumbar puncture.

Conclusions: Perfluorocarbon could enter the cranial space during ocular surgery and threaten the life of patient. Further migrating through subarachnoid space provide the opportunity to remove it.

\section{Background}

Perfluorocarbon was reported earlier before for treating traumatic retinal detachments[1], and it has been widely used since 1987. Perfluorocarbon liquids can compress and disorganize the retina and are used in a variety of ocular surgeries. Usually, because of the toxicity, residual perfluorocarbon is removed immediately after application and should not be seen in the radiology exams[2, 3]. Perfluorocarbon has barely been reported to enter the central nervous system. David R[4] reported a case of cranial perfluorocarbon, which for the first time, described the migration of perfluorocarbon in the cranial space. Here we reported a patient with perfluorocarbon migrating into subarachnoid and removing by lumbar puncture.

\section{Case Presentation}

A 65-year man was admitted to hospital because of a severe injury on the left eye. The man was hit by a rock one hour prior to the admission. Entering visual acuity was 20/63 OD and very poor on the left side. Examination showed left conjunctival congestion and swelling, corneal swelling, anterior chamber hematoma. The left pupil was deformed and dilated without a change in the levels of light. Lens was dislocated into vitreous chamber. Blood was seen in the vitreous body. Fundus was not seen clearly. The patient's medical history was unremarkable. Preoperative laboratory tests were unremarkable. 'Ultrasonic lens fragmentation+posterior vitrectomy+perfluorocarbon liquid instillation+photocoagulation+fluid-gas exchange' was performed to fix his left eye injury. In the surgery, $5 \mathrm{ml}$ perfluorocarbon liquid was instilled. Right after the fluid-gas exchange, oxygen saturation and blood pressure dropped abruptly, and the patient was unconscious. The ophthalmologist closed the incision immediately. Epinephrine $(1 \mathrm{mg})$ and rapid fluid replacement were given immediately. After emergent intratracheal intubation, the patient was transferred to the intensive care unit (ICU) for further treatment. Despite 100\% oxygen supplement and fluid resuscitation, the blood pressure and oxygen saturation failed to improve. Norepinephrine 
(1.0ug/ $\left.\mathrm{kg}{ }^{\star} \mathrm{min}\right)$ was then pumped to maintain blood pressure. The positive end-expiratory pressure on the ventilator was set as $15 \mathrm{mmHg}$, arterial oxygen partial pressure/fractional inspired oxygen (PO2/FiO2) was 40 . Brain natriuretic peptide, creatine kinase-MB, troponin and electrocardiograph was normal. Bedside ultrasound showed no "D" sign, existence of pleural sliding and EF was $68 \%$. Thoracic X-Ray showed effusion in both lungs(Figure1). Therefore, veno-venous extracorporeal membrane oxygenation(V-V ECMO) was performed to maintain blood pressure and oxygen saturation. Head CT showed hyperdense aggregation around the optic nerve and in the cerebellum (Figure 2A and $\mathrm{B}$ ). The radiodensity of the aggregates was $550 \mathrm{HU}$. Therefore, the aggregates were suspected to be the perfluorocarbon. The patient recovered gradually, and ECMO was discontinued four days later. Then the patient was encouraged to keep an upright position to facilitate the downward flow of perfluorocarbon (density $1.75 \mathrm{~g} / \mathrm{ml}$ ). Seven days after the surgery, a CT scan showed perfluorocarbon was at the thoracic vertebra level (Figure 2C). On day twenty-one, it arrived at the level of lumbar cisterna (Figure 2D). Lumbar puncture was then performed on day twenty-three. During the puncturing, lower extremities, head, and chest were raised to make the puncture site lowest. The patient was asked to keep either left or right lateral decubitus position. Finally, $4 \mathrm{ml}$ perfluorocarbon was extracted (Figure 3 ). After treatment, the patient had left vision loss, but other symptoms fully recovered.

\section{Discussion}

The patient with no known cardiac or pulmonary abnormality experienced a sudden decrease in oxygen saturation and blood pressure during eye surgery, indicating causes other than the cardiac or pulmonary origin. Besides, this patient had no skin rash, fluid resuscitation and epinephrine failed to improve the symptoms, which was not very support anaphylactic shock. Since the symptoms initiated during the ocular surgery, and with the help of radiology exams, we believed that perfluorocarbon could potentially enter the cranial space through the optic nerve sheath and then gradually increased intracranial pressure. These together might trigger neurogenic pulmonary edema and neurogenic shock. In reasonable condition, blood-retinal barrier and blood-optic nerve barrier could separate optic nerve sheath with subarachnoid space. However, blunt trauma could have damaged the integrity of the blood-retinal barrier and further induce the communication between optic nerve sheath and subarachnoid space[4]. In our case, the patient's eye trauma might injure the blood-retinal barrier, and even the optic nerve sheath. With the increased intraocular pressure, perfluorocarbon might spontaneously circulate through optic nerve sheath to subarachnoid space, which change the intracranial pressure. ECMO was necessary to improve the patient's oxygenation and hemodynamics. In the end, the perfluorocarbon could be accessible by lumbar puncture.

According to our case, a great amount of perfluorocarbon migrating into subarachnoid space could disrupt medullary autonomic activities. If neurologic signs, such as abrupt onset hypotension, hypoxemia, irregular respiration, or intracranial hypertension develops after perfluorocarbon application, perfluorocarbon leakage should be highly suspected. We propose that lumbar puncture is a possible way to remove partial perfluorocarbon. 


\section{Conclusion}

Perfluorocarbon could enter the cranial space during ocular surgery and further migrate through subarachnoid space. It is crucial to discover the complication and make a treatment. We propose using lumbar puncture to remove perfluorocarbon after its sinking to lumbar cisterna by gravity.

\section{Declarations}

\section{Acknowledgments}

The patient kindly gave full written permission for this report to be made including the use of images.

Affiliations:Department of Intensive Care Unit, Jinhua Municipal Central Hospital(Jinhua Hospital of Zhejiang University)

\section{Corresponding author}

Correspondence to Hongying $\mathrm{Ni}$

\section{Authors' contributions}

Hongying Ni was responsible for the ongoing care of the patient in hospital. Zhongheng Zhang and Qing Wang conceived of the case report. Lin Chen researched and drafted the manuscript. All authors read and approved the final manuscript.

\section{Availability of data and materials}

Reasonable requests for data and materials will be considered and should be

made in writing to the corresponding author.

\section{Consent to publication}

We have obtained written consent from the patients and their relatives already.

\section{Disclosure of interest}

The authors report no conflict of interest.

\section{Ethics approval and consent to participate}

The ethics committee of the hospital approved the case study.

\section{Funding}

None. 


\section{References}

1.Chang S, Reppucci V, Zimmerman NJ, Heinemann MH, Coleman DJ. Perfluorocarbon liquids in the management of traumatic retinal detachments. OPHTHALMOLOGY 1989, 96(6):785-791, 791-792.

2.Christoforidis JB, Caruso PA, Curtin HD, Fiore T, D'Amico DJ. CT characteristics of intraocular perfluoroN-octane. AJNR Am J Neuroradiol 2003, 24(9):1769-1771.

3.Liu W, Gao M, Liang X. Management of Subfoveal Perfluorocarbon Liquid: A Review. OPHTHALMOLOGICA 2018, 240(1):1-7.

4.Malin DR, Aulino JM, Recchia FM. Intracranial hyperdense subarachnoid perfluorocarbon droplets after attempted retinal detachment treatment. Emerg Radiol 2007, 14(4):261-263.

\section{Figures}




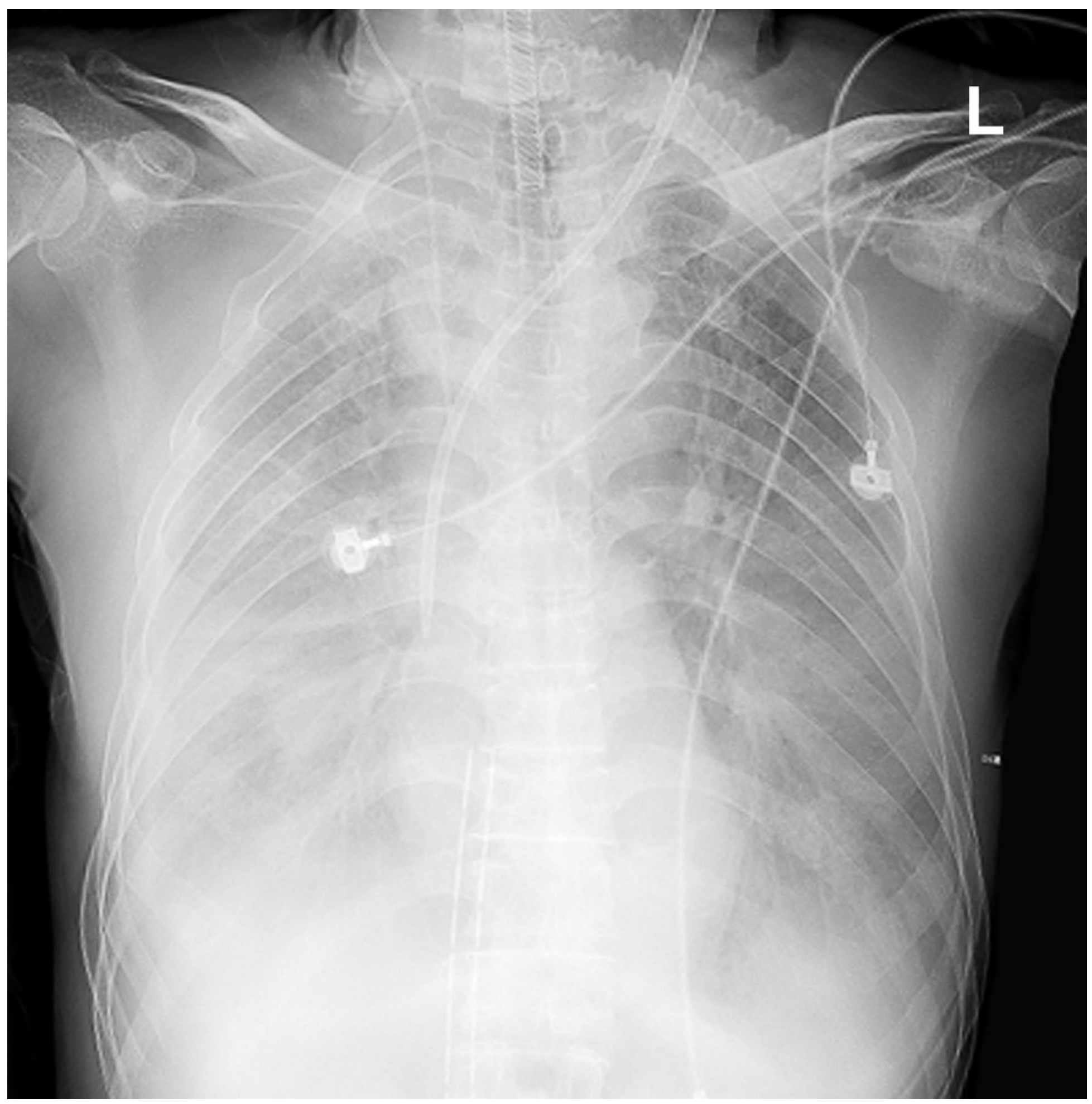

Figure 2

Thoracic X-ray shows Effusion in both lungs. 

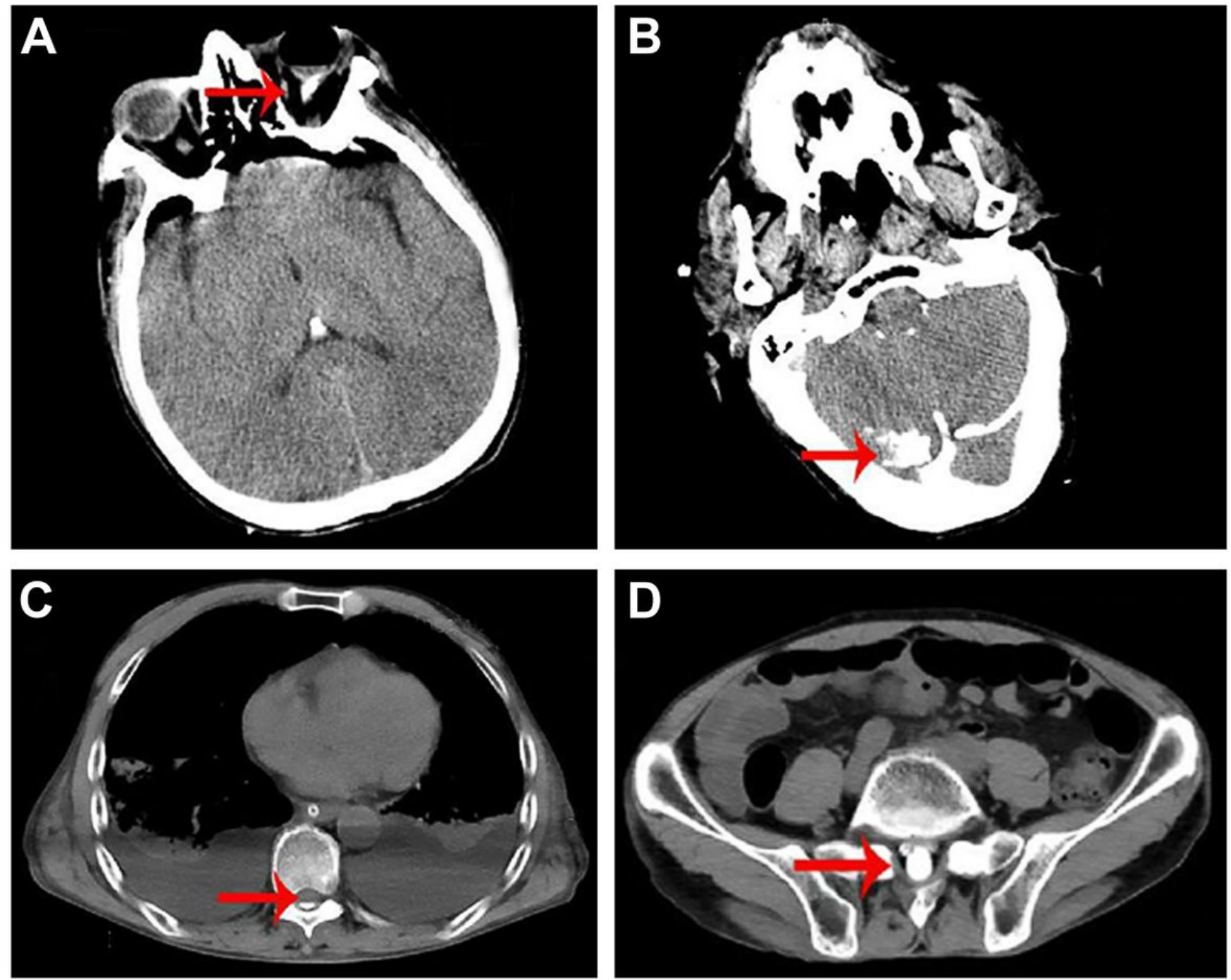

Figure 3

Head computed tomography shows hyperdense aggregation around the optic nerve; 2B, 2C,2D: High density(red arrow) shadow of central nervous system. 


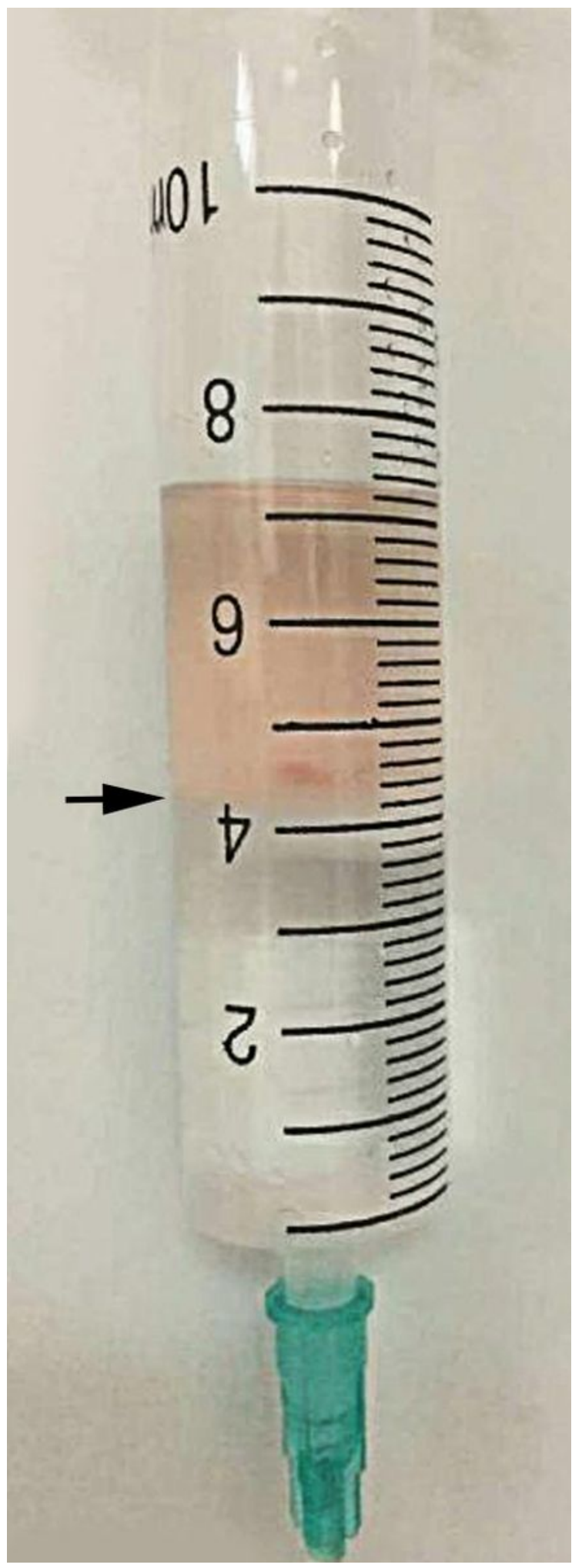

Figure 5

Stratification of perfluorocarbon and cerebrospinal fluid. 